\title{
2 The biographies of artifacts and practices methodology for the study of sociotechnical change
}

\subsection{Introduction: facing the inconvenience of sociotechnical change in social analysis ${ }^{1}$}

A considerable part of sociotechnical change is inconveniently structured for social scientists. The settings and practices that shape technology are complex and intricate, making them laborious to investigate compared to more commonplace areas of social inquiry, such as studying interactions, attitudes, or institutions and organizations. This has pushed many scholars of the social study of technology toward intensive ethnographic engagements with particular contexts. This particularly concerns the many types of user contributions to sociotechnical change outlined in the introductory chapter, as they tend to fall within the expanse of the invisible work related to science and technology making (Strauss and Star, 1999; Kohtala et al., 2020).

While the intensive ethnographies have proven to be an important strategy for an adequate understanding of sociotechnical change, it has been recognized that many processes of sociotechnical change occur over years and decades and across a range of contexts. New technologies are shaped in multiple interlinked settings and processes characterized by high contingencies and many different choices. The analytical templates needed to cover such broad expanses of time and space have, by default, resorted to coarser "granular" analysis and description. This means that the study set-up-from the outset-brackets out the underlying complexity and intricacy of phenomena. The net result has been the "hollow" or "smoothed" description and analysis of phenomena: a complex phenomenon is noted and may even be used as an explanatory factor, but its actual realization or the dynamics within it are not attended to adequately (Miettinen, 2003; Hyysalo, 2009; Scott-Kemmis and Bell, 2010).

These two key insights - the need for intensity and the expansion of analysis-emerged as early as the 1980s and 1990s in studies of the social studies of technology (SST) through the separate research programs of ethnographers, sociologists, and historians of technology (McKenzie and Wajcman, 1998; Williams and Edge, 1996). Yet their combined effect continues to hold an inconvenient methodological truth for the social study of technology: to account for the shaping of technology, one would need to study the wide

DOI: $10.4324 / 9781003133919$ 
range of settings and the interlinkages between them, as well as the intricate practices therein (Williams and Edge, 1996). It would not be enough to posit that a framework would need to study both actors and structures, and stability and change (e.g., Bijker, 1995; Geels et al., 2016); one must also study these and their interrelations together.

The inconvenience is that realizing the above ambition is much easier said than done. The early technology studies' understanding of sociotechnical change emerged from a patchwork of ethnographic and historical studies that either focused deeply on one or a few moments and sites or, alternatively, sought a broad-brush overview of technology development with a few illustrative deeper examples added (for more detailed critique, see Russell and Williams, 2002; Hyysalo, 2010). This inconvenience became further aggravated in the 2000 s when the "mark 2" social shaping of technology studies discovered the importance of technology-in-use (see Chapter 1) and the importance of social movements and other stakeholder groups in the shaping of technology (Sørensen and Williams, 2002; Williams et al., 2005), thus adding to the array of relevant settings to be studied in-depth and then connected in order to produce an understanding of the dynamics of technological change (Pollock and Williams, 2008, 2016; Stewart and Hyysalo, 2008).

\subsection{Framing effects and a premature sense of closure}

The result was a methodological paradox. SST had produced an understanding of technological change that was arguably more sophisticated and grounded than that provided by mainstream innovation studies - yet it was one that would require research designs that were at odds with SSTs own study templates.

The gravity of these "framing" or "closure" effects was brought to the fore in the 2000s as researchers sought more complex research designs to remedy the paradox (Stewart and Williams, 2005; Pollock and Williams, 2008; Hyysalo, 2010). These research designs repeatedly indicated that studying any given moment and site of innovation would give a significantly different picture of the agency, structure, impact, and materialities related to the technology under study.

In this light, many fellow researchers, and previous studies by the author as well, have fallen victim to a false sense of "natural closure" when conducting their studies, believing there was little point in extending studies to new settings and levels of analysis. Another way to put this is that a premature sense of theoretical saturation (Glaser and Strauss, 1967) is common when researching sociotechnical phenomena.

From the variety of potential closure effects, the first to gain attention became conceptualized as "snapshot bias" (Pollock and Williams, 2008). For instance, when the design of new technology was studied, users were found to be actively configured (see, e.g., Woolgar, 1991; Oudshoorn et al., 2004). However, when technology was followed into use, the users would, in turn, be found to reconfigure (or domesticate) both the technology and the designers (Mackay et al., 
2000). Further on, episodes of the co-configuration of technologies, designers, and users were discovered once the research design was extended to cover these events (Hyysalo, 2004, 2010; Pollock and Williams, 2008). In all, such studies comprised an expanded approach to study the "biographies of artifacts" and their varying shapes (Kopytoff, 1986) across a usually great variety of settings and temporal time frames. In other words, we find that not only are methods performative (Law, 2007) but core issues in research design, such as choosing the site and time of the study, could prefigure which kind of sociotechnical shaping processes one would be disposed to find and, importantly, which ones one would be very unlikely to encounter (Hyysalo, 2010).

The second framing effect concerned the granularity of data and analysis (Hyysalo, 2010) and is in this book is called hollow-arch bias. We define hollowarch bias as a portrayal of a sociotechnical phenomenon with a high level of abstraction that neglects some of the underlying mechanisms that question or invalidate parts of the high-level construction. The constructs appear solid until one digs beneath their empirical surface.

Hollow arch does not mean "a house built on sand" or "a house of cards," as in having no empirical backing for theory constructs at all-there is some backing, yet it is of a limited and superficial kind. And this is part of the problem: as there is some support, scholars who prefer to operate at the broad systems level continue to take for granted and use the hollow constructs and therefore do not complicate their work with more complex and research-wise more inconvenient findings and theoretical constructs on which more focused studies insist.

A prominent example of a hollow-arch construct is that of post-implementation learning effects, known as learning by doing, using, and interacting (Rosenberg, 1979, 1982; Lundvall, 1988). The continued improvements in manufacturing efficiency (learning by doing), the usage and development of tools (learning-by-using), and interactions between producers and users (learning by interacting) appeared straightforward when changes from one product version to another were compared (e.g., Arrow, 1962; Gardiner and Rothwell, 1985) and then taken as pillars for various innovation system constructs (e.g., Lundvall, 1988; Lundvall \& Vinding, 2005). But once these learning processes were studied in more detail, most of the "learning" in these conceptions turned out not to be learning at all as the outcome changes had resulted from changes that happened during the actions, organizations, interactions, arrangements, designs, and materials used, often without recourse to possible learning at the manufacturing and user sites (von Hippel and Tyre, 1995; Scott-Kemmis and Bell, 2010; Nielsen, 2016; Miettinen, 2003). Just as importantly, when the learning that was taking place was studied in detail, it turned not to be as straightforward as depicted by the learning by doing, using, and interacting model (Lundvall, 1988). Instead, the learning processes and outcomes were found to be haphazard, vulnerable, conflictual, and required high amounts of work to coordinate between parties (Hasu, 2001; Hyysalo and Lehenkari, 2002; Hyysalo, 2006, 2009; Hyysalo and Hakkarainen, 2014). 
The point is this: also in other sociotechnical phenomena, high grain-size materials have a tendency to produce hollow-arch descriptions. They may be amenable to neat explanations while overlooking the details of the underlying phenomena that may substantively invalidate the theory constructs laid on top of them. If, and oftentimes as, the more fine-grained studies do not produce similar aggregate level data than that on which the hollow-arch constructs are built on, the concerns raised remain conveniently ignored. The bias resulting from hollow-arch and snap-shot study templates is thus similar regarding the false sense of adequacy in explaining sociotechnical phenomena, even though the mechanism by which the 'blinkering' occurs is very different: one results from an overemphasis on particular study types and sites, and the other from an over-zealous abstraction.

The knowledge gained from research programs that combined both intensive and expansive studies gradually lead to the articulation of a longitudinal multi-site research approach called the "biographies of artifacts and practices" (BOAP) approach. This perspective has evolved from its onset in the early 2000s to a point today where some 30 long-term studies have been conducted with it.

\subsection{The BOAP approach: key characteristics}

The BOAP approach is a methodological approach to the study of sociotechnical change that-even though it has its roots in research informed by broader theoretical approaches of the social shaping of technology (mark 2), symbolic interactionist S\&TS, and activity theory-has purposefully been partially detached from these theories and rendered compatible with several substantive traditions in the S\&TS field and innovation process studies.

BOAP studies feature eight recurring characteristics, which can be considered core markers of the approach. Most BOAP studies feature a varying subset of these methodological responses to common contingencies and differences in the settings of sociotechnical change, in other words they do not present a formula to be applied in all research. The contingencies of sociotechnical phenomena make it difficult for researchers to reliably predict in advance (for instance, on the basis of theory) what might be revealed and occluded by selecting a singular vantage point or a limited set of vantage points, and each of the BOAP characteristics has arisen to handle this challenge. The common characteristics are as follows:

1. Spatial and temporal reach that is sufficient to empirically engage the dynamics of the studied phenomenon (e.g., studies could look at an individual innovation together with the evolution of an industrial field). BOAP studies encompass multiple loci and times wherein sociotechnical change is shaped and moves beyond singular "snapshot" accounts. This is in line with the ambition of multi-sited ethnography to go beyond particular organizational settings, being particularly relevant to the highly dispersed 
processes of scientific and technological life (Hine, 2007; Monteiro et al., 2013; Silvast and Virtanen, 2019; Marcus, 1995) and by those advocating more structural considerations as part of S\&TS analyses (e.g., Klein and Kleinman, 2002; Russel, 1986; Silvast et al., 2013).

2. The shaping of technology and practices is analyzed as taking place within ecologies of interconnected actors. This means not only studying the actors with respect to how they affect the studied technology (such as in Bijker's [1995] "relevant social groups"), which leaves aside the rationales by which they operate and often also the complex and subtle additional mechanisms by which actors relate (Hyysalo, 2010; Pollock and Williams, 2016). This BOAP premise bears a close similarity to those developed in, for example, "linked ecologies" (Abbott, 2005), the "social worlds/ arenas" framework (Star and Clarke, 2003), and studies of the "networks of activity systems" (Engeström, 2000).

3. It may be particularly fruitful to identify and research interstices, the moments and sites in which the various focal actors in the ecology interlink and affect each other and the evolving technology. An overall understanding of the ecology of actors is typically used to pinpoint key locales where these interstices may be researched in detail. The focus on interstices is shared by many in S\&TS, and the use of broader-scale analysis to identify the sites to focus on is found in studies of infrastructures (Monteiro et al., 2013; Ribes and Polk, 2015) and in studies examining the evolution of scientific fields (e.g., Cambrosio and Keating, 1995; Edwards, 2010; Fujimura, 1996).

4. Research is pursued at multiple temporal and spatial scales. The BOAP studies that are at odds with accounts that assume sociotechnical change could be adequately understood through "bird's-eye" descriptions only. There is a need to bridge the analyst's bird's eye view and the actors' real-time "frog's-eye" perceptions, which typically feature high levels of uncertainty and contingencies (e.g., the "fog of innovation" [Höyssä and Hyysalo, 2009]) that can entirely disappear from historical data and broad overviews. Hollow-arch biases and other data granularity related biases are discovered in BOAP investigations time and again. Questioning the dominant research framings in literature can be the starting point for an inquiry into a richer set of contexts (e.g., Stewart and Hyysalo, 2008) or the major outcome of the investigation (e.g., Hyysalo, 2010; Pollock and Williams, 2008, 2016). Multiple-scale research designs can be found elsewhere as well, for instance, in technology and organization studies, in studies of practices (e.g., Nicolini, 2012), in activity theory (Cole, 1996; Engeström, 2000), and in the symbolic interactionist social worlds/arenas framework (Strauss, 1978; Becker, 1982; Clarke and Star, 2003; Clarke, 2005).

5. Different temporalities and spans of change are seen as multiple enacted contexts (Hyysalo, 2010), not as the ontologically distinct layers that are presumed to exist, for example, in the multi-level perspective (Braudel, 
1995; Geels, 2002; Geels and Schot, 2007) or the traditional sociological approaches to the context that locate action within a context conceived as "surrounding layers" (Strauss and Corbin, 1998). In BOAP, events are seen as simultaneously constituting and being constituted by broader patterns: the context for any situation is understood as being comprised of differently paced constituents, as previously discussed in microhistory (e.g., Levi, 1988) and socio-cultural psychology (Cole, 1996; Engeström, 1987), and in distributed cognition (e.g., through the "Hutchins' cube" where the same moment is analyzed in terms of the development of practitioners, practices, and the situated enactment of action [Hutchins, 1995]). BOAP thus seeks to inquire into the links between relevant constituents in order to see their influences and interrelations (or the lack thereof). Studying different contextual constituents means employing an array of often differing conceptual tools, analysis types, and methods in diverse materials (Hyysalo, 2010: 43). BOAP's preference for ethnographic study thus does not mean an in-built "micro-sociological" focus but an examination of how the structuring elements are present in real-life situations and, in turn, how the situations re-shape the structuring elements and what can be learned about the patterns and structures as they are enacted. The position resonates with Situational Analysis by Clarke (2005), yet refrains from flattening the empirically salient topologies in contextual factors (Star, 1996; Akera, 2007) and thus differs markedly from actor-network theory (Latour, 1987, 2005) or ethnomethodology (e.g., Suchman, 1987).

6. Akin to many STS approaches, BOAP studies insist on paying attention to materiality: the content and form of technology as it shapes, and is shaped by, the interrelations between actors (Latour, 2005; Kallinikos, 2004). This goes for the material nature of the focal technology studied (and differences that results from these being, for example, energy technologies that span several domains of hardware and software vs being easily modifiable physical objects), as well as the production systems, tools, and infrastructures that designers and users use to shape them (cf. Cambrosio and Keating, 1995; Galison, 1997). This is to say, BOAP insists on carefully investigating the different materialities and their effects in different sites and times of a technology's life and carefully reflecting on what this entails for the overall research design-something more often claimed than carefully done in social studies of technology.

7. Through the above, BOAP studies seek balanced and empirically adequate accounts of what different actors do rather than assume, for instance, that key design decisions must have been made by designers (for, as we discuss below, they may be made by users).

8. The detailed dynamics of sociotechnical change are attended to, both empirically and theoretically. This has been the focal interest in all BOAP research to date. It has involved pursuing a detailed understanding of change in different settings and moments. This is at odds with resorting to high-level 
depictions of sociotechnical change. We discuss below the risk that widely adopted conceptions of studying sociotechnical change, such as social construction, mutual shaping, or systems transition, used as a template to characterize the relevant processes and net outcomes, may be used as an excuse for only using high-level generalization and occluding the detailed processes that factually constitute it, often against a declared intention to do so (for example, Bijker, 1995; Schot and Geels, 2007; Geels et al., 2016).

\subsection{Ecologies of actors and their interstices: theory bridging as the ninth BOAP characteristic}

The notion of ecologies of actors in the present use of BOAP is, in this book, rooted in a wider "ecological" view of sociotechnical relations, typically informed by Chicago sociology and the ensuing institutional analyses of professions and symbolic interactionist research on work and technology (e.g., Strauss, 1978, 1993; Star, 1995; Abbott, 2005; Clarke and Star, 2003; Akera, 2007). Abbott (2005: 248-249) provides a set of particularly clear articulations of the position of ecological sociology. In respect to broader social theory he remarks:

When we call a set of social relations an ecology, we mean that it is best understood in terms of interactions between multiple elements that are neither fully constrained nor fully independent. We thus contrast ecology with mechanism and organism on the one hand and with atomism and reductionism on the other. The latter contrast is straightforward and general: ecology involves some kind of relation between units whereas atomism and reductionism involve only qualities of units themselves or of their aggregates. With mechanism and organism, the contrast is more specific. When we encounter complete and routine integration in the social world, we employ the metaphor of mechanics, as in the "rule-governed systems" of role theory, for example. When we encounter systems whose elements move together in flexible homeostasis, we use the metaphor of organism, as in structural functionalism. By contrast with these two, in ecological thinking, the elements are not thought to move together at all; rather, they constrain or contest each other. "Ecology" thus names a social structure that is less unified than a machine or an organism, but that is considerably more unified than is a social world made up of the autonomous, atomic beings of classical liberalism or the probabilistically interacting rational actors of microeconomics.

In the current theoretical landscape, an ecological view of social phenomena finds close parallels in STS conceptualizations that acknowledge that complex, large-scale changes involve not only "hot" sociotechnical relations, in which the nature of actors is uncertain and their relations are fluctuating, and "cold" sociotechnical relations, where technology characteristics, markets, institutions 
and organizations have already congealed into stable and distinct entities (Callon, 1998), but also, and predominantly, varying topologies that feature mixtures of the two (Star, 1995, 1996; Jørgensen, 2012; Hyysalo et al., 2019a). In such a view, functionalist system conceptualizations should be heralded for their insistence on the remarkable power and obduracy of solidified sociotechnical structures, and the corresponding difficulties of system change (see, e.g., Hoogma et al., 2002; Geels, 2002). Again turning to Abbott: "[n]o social world ever exists without a pre-existing topology of some sort" (Abbott, 2005: 249). At the same time, the systems-oriented concepts are seen to remain deficient in understanding highly dynamic and emergent processes because they lack the means to address the various forms of agency and interplay between actors who negotiate the spaces and timings of the processes of far finer granularity (but no less complexity) that comprise whatever is realized of the change (Garud and Gehman, 2012; Spinardi and Slayton, 2015).

Conversely, theorizing that rests on actor networks as ever-expanding sets of overflows without consideration of pre-existing obdurate framings is seen to lapse toward sociotechnical voluntarism. Amid their socio-material assemblages, humans and other entities "make their own histories, but- to modify the Marxian dictum-in that making they produce larger structures that in turn render them unable to make those histories under conditions of their own choosing" (Abbott, 2005: 254).

Thus, in theories popular in SST and innovation studies this epistemological stance is different to both ANT (Latour, 1987, 2005) or, for instance, the MLP (Geels, 2002; Geels and Schot, 2007). ANT progresses from actants and the networks they form, leaving all issues of topology and structuration to be empirically settled, and it does not have pre-existing or generic conceptual registers to address topologies and structures (i.e., it relies on what has come to be called "flat" ontology). The MLP, in turn, presupposes a pre-defined structural ontology that study findings have to populate. In contrast, ecological views acknowledge the existence of previously identified social entities as sensitizing concepts that orient empirical inquiry towards topologies and ecologies, and suggest empirical points of entry but do not assume that these necessarily take a pre-defined structure or shape and thus leave the outcome analysis of both ecologies and topologies to be empirically built for the topic at hand, not structurally pre-determined (Strauss, 1993; Star and Clarke, 2003; Akera, 2007).

To understand this view better, we elaborate on some of the key concepts in more detail.

Ecologies of actors result in mutually defining lines of action by the actors involved in an event (Blumer, 1969; Strauss, 1993) and the patterning of events that have resulted in more durable social institutions and the topologies of power, resources, skills, constituencies, and commitments that have resulted from these (Blumer, 1969; Strauss, 1993; Becker, 1982; Clarke and Star, 2003). Ecologies of actors are typically populated by a range of differing sociotechnical entities, some of which are nested and others which are not, or which are only partially nested. Organizations, social movements, 
electoral constituencies, professional and industry associations, science labs, start-up companies, families, and governmental agencies all have distinct characteristics and therefore different capacities for action. Regarding an event or an arena, the people in these sociotechnical entities are typically aware of each other and the patterns of previous actions (Becker, 1982, 1998). They also tend to have complex interrelations that reach, in time and space, beyond a single event or arena (Star, 1989a). The above has a propensity to result in many-to-many translations that extend over time rather than being one-time contestations or translations of interests (Star, 1989b; Bowker and Star, 1999).

Arenas for social action refer to sociotechnically constituted sites rather than simple geographic locations, wherein the current and renewed order between actors is negotiated (Clarke and Star, 2003). Arenas should be seen as settings that feature some measure of stability and recognizability for the actors involved in them. Arenas can be at various stages of formation, ranging from the emergent and fluid networks that are well depicted by the "arenas of development" concept (Jørgensen, 2012) to established arenas that feature pre-existing sets of (bundled) issues and rules, and require certain skills, resources, and materials in order for there to be competent action.

Peripheral participation and multiple memberships are commonly recognized in the study of sociotechnical phenomena. Social life is seen to be made out of events, and stability ensues from the gradual formation of boundaries leading to entities, rather than entities already coming with boundaries (Abbot, 1995). This means that social entities — such as arenas, social worlds, or organizations - are seen to have "porous boundaries" and varying centrality of membership. Social formations may invest in guarding their membership, but people, objects, and infrastructures hold membership in multiple social worlds by default (Strauss, 1993; Clarke, 1998; Bowker and Star, 1999). Some such memberships result in go-betweens, others in boundary spanning, and yet others transform one or several social entities via mutual influences (Star, 1989b; Becker, 1982, 1998).

Given that ecologies of actors present very complex and rich research terrains, the focalization of research on particular sets of issues and actor groups is a practical necessity. For instance, Abbott's studies of the linked ecologies of professions are focalized on major and minor professions, the turfs they occupy in society, their emergence and waning, and the processes and results of negotiated order between them (Abbott, 1988, 2005). Clarke's study of reproductive medicine focuses on the interactive developments between key social worlds, the arenas between them, research programs, and the laboratories and instrumentations involved (Clarke, 1998, 2005). Star and Strauss (1999), in turn, focused on the invisible work inside research laboratories. The focalizations can be of different intensity; the study of invisible work is a more fine-grained focalization than that of Clarke, which in turn is more fine-grained than that the analysis of changing institutions by Abbott, yet they all expand out to the relevant ecologies of actors and processes therein in order to make sense of their focal interests. 
The focalization leads us to articulate a ninth BOAP guidepost that is particularly salient in the present line of investigation, namely:

9. Theory and the research tradition cross-linking. BOAP investigations typically mean deploying a number of mutually complementary studies on different aspects of the biography of technology, and over different time frames of analysis. Each focalization is typically relevant for an existing body of research and theory building. A common characteristic of a BOAP investigation is that its sub-study designs tend to be informed by the research templates and theories relevant to the sub-study, but these become linked and are critically compared within the scope of the overall BOAP investigation. The ensuing cross-linking and qualifying insights are pursued between different detailed foci (and literature), as well as between the detailed sub-studies and broader but more coarse grain-size analyses and theory framings (Pollock and Williams, 2008; Hyysalo, 2010), as well as varying fluidity of the social settings and structures studied. Such a strategy is neither naively inductive nor based on the testing of hypothesized theoretical models but can navigate between the two (Hyysalo et al., 2019).

To concretize: in the present book we began with a user-innovation focus and the research framing that this research community has established (Hyysalo et al., 2013a, 2017; Mattinen et al., 2014). We did not just reproduce it though; we purposefully gathered data on related actors, dynamics, and interstices in our ethnographies and interviews. This next led us to do a study on the embeddedness of user innovation in internet peer communities using an S\&TS framing for knowledge infrastructures (Hyysalo, 2013b); a comparison of ownership and technical configurations (Juntunen and Hyysalo, 2015); the adoption of renewables using a domestication framework (Heiskanen et al., 2014; Juntunen, 2014b); the internet peer communities influence to the overall diffusion and transition process (Hyysalo et al., 2018); historical analysis of heat pumps in Finland using a transition framing (Lauttamäki and Hyysalo, 2019); and finally, we connected the sub-studies in an overview study of users in a heat-pump transition (Chapter 5). All these sub-studies are cross-linked in the present overall volume of this BOAP investigation.

\subsection{Methodologically speaking: coverage of sociotechnical phenomena by analysis unit, observation unit, and data}

The closure effects and core markers discussed above have been subject to discussion and debate for two decades now. As such, it has become evident that there are now communities of researchers who have experienced first-hand the framing effects through studying the same technology in multiple settings and recognizing the importance of this issue. But it has become equally clear that others - perhaps those without a personal experiential basis - tend to go to considerable lengths to find alternative explanations for the study design's 
framing effects or to simply seek to avoid the issue altogether. This being the case, let us articulate the issue with a degree of formality through examining the relationship between analysis units and observation units in different strands of innovation studies and technology studies and the BOAP approach.

\subsubsection{Analysis units, observation units, and data in studies of technology adoption and innovation}

A good entry point for examining the analysis and observation units in innovation studies is to examine survey-based studies of innovation. These have readily graspable analysis units, observation units, and data, and in addition they continue to be widely deployed (in fact, well beyond the application area that their study set-up permits, as demonstrated below). Survey-based innovation studies can be exemplified by the OECD's community innovation survey and innovation diffusion survey. In both surveys types, the set-up is similar: the analysis unit is the adoption of innovation (the diffusion of innovation) or the introduction of innovation (a community innovation survey). The observation unit in both is the survey response to a set of questions based around "Have you adopted a particular innovation?" or "Have you introduced an innovation?" Insofar as the entity to whom this question has been directed is the person who answers the survey and the invention is discreet and clearly recognized, there is strong coverage between the phenomenon studied (the adoption/introduction of the invention by the person or company), the analysis unit by which it is methodologically operationalized (the adoption/introduction of novelties as reported by the respondent), and the observation unit, which the real-world counterpart to the analysis unit (a response to the survey question on whether or not one has adopted/introduced a novelty). The survey does not necessarily give very rich or detailed data on the observation unit but gives sufficient data for the purpose at hand. Figure 2.1a illustrates this with respect to a diffusion survey.

However, the coverage between the phenomenon, the analysis unit, and the observation unit changes if the knowledge interest concerns a more complex entity, such as a community or an organization. In Figure 2.1b, we see

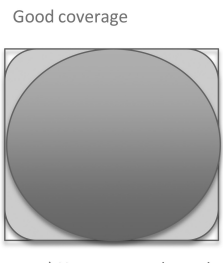

a) Has person adopted

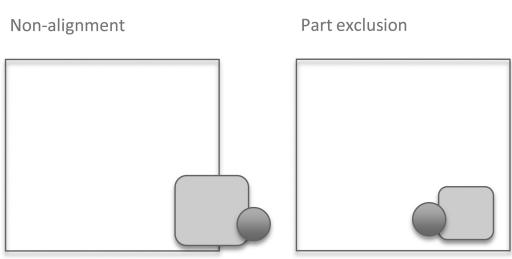

b) Has somebody adopted

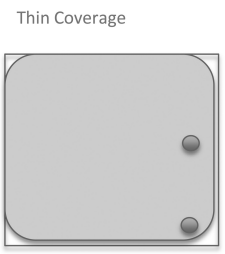

d)Organizational adoption

Figure 2.1 Coverage between the analysis unit, observation unit, and data in technology adoption research. 
how the situation changes if the respondent to the survey answers on behalf of others without first-hand certainty of what exactly has happened, the default case in any organization or community of more than 50 people. The individual respondent is assumed to somehow be able to speak for the whole organization, and the research takes the organization as the unit of analysis without considering differences of access to information and different orientations within the organization that affect its observation unit.

The situation is only slightly improved if, say, two respondents in an organization of 50 employees answer the survey regarding the typical 1-3-year radius used in these surveys. The analysis unit, the adopting/introducing organization, is now covered by an observation unit that is aligned, but the coverage is rather thin and easily amenable to the hollow-arch bias discussed above (Figure 2.1d). These are well-known weaknesses of diffusion studies regarding organizational diffusion (Rogers, 2010) and apply to survey-based innovation studies as well.

If the novel item is ambiguous or can be decomposed, added onto, or integrated in any way (Figure 2.1c) the analysis unit of "adoption" and its relation to the observation unit becomes undifferentiated-respondents can interpret adoption as only straight adoption or also as partial, piecemeal, and innovative adoption and answer accordingly (Kohtala et al. 2020). This is an equally wellknown problem in diffusion research (Rogers, 2010; Helminen et al., 2018), and, as we discuss in Chapter 3, it also features in user-innovation research done using surveys resulting in propensity to downplay the amount of local modifications and adaptations by users.

When thin coverage of organizational adoption by the observation unit is combined with an undifferentiated analysis unit in regard to adoption/adaptation, the reliability (as well as the validity) of the results is low regarding the phenomenon under study (Rogers, 2010). In research on the organizational adoption of complex technologies, such as software products, such a lack of fit has been remedied by in-depth interview and ethnographic studies. These allow for the use of a large series of observation units, covered by rich data, with the help of which issues such as adoption and adaptation (and over 20 other adoption moves) can be identified and reliably studied (DeSanctis and Poole, 1994; see Figure 2.2a).

While richer data and more numerous observation units add reliability, they also prompt researchers to ask new questions. The improved coverage allows moving from the question of whether or not adoption or adaptation has happened onto questions about how it happened and what happens in adoption. This shift took place in the 1990s (Silverstone, 1992; Berger et al., 2006; DeSanctis and Poole, 1994; McLaughlin et al., 1999) and found its parallel in studies of innovation, which moved beyond asking if and what, for instance, users or producers innovate, moving onto a processual understanding of how their innovation happens (van de Ven, 1999; Russel and Wiliams, 2002).

But changing the question resets the requirements for coverage. Answering these new questions adequately requires more fine-grained units of analysis and the corresponding observation units and data to cover them. The same 


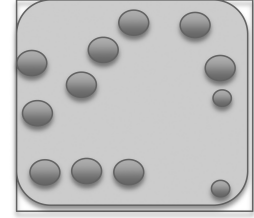

a) Adoption by orgnization

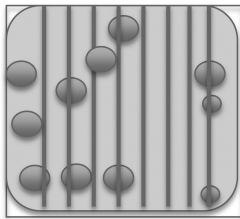

b) Adoption process

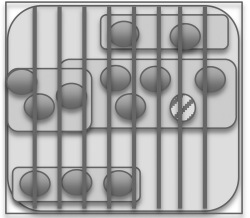

c) Adoption process and sub-processes

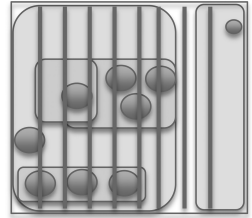

d) Implementation and impact studies of IT adoption

Figure 2.2 The analysis units and observation units in more in-depth studies of innovation and organization.

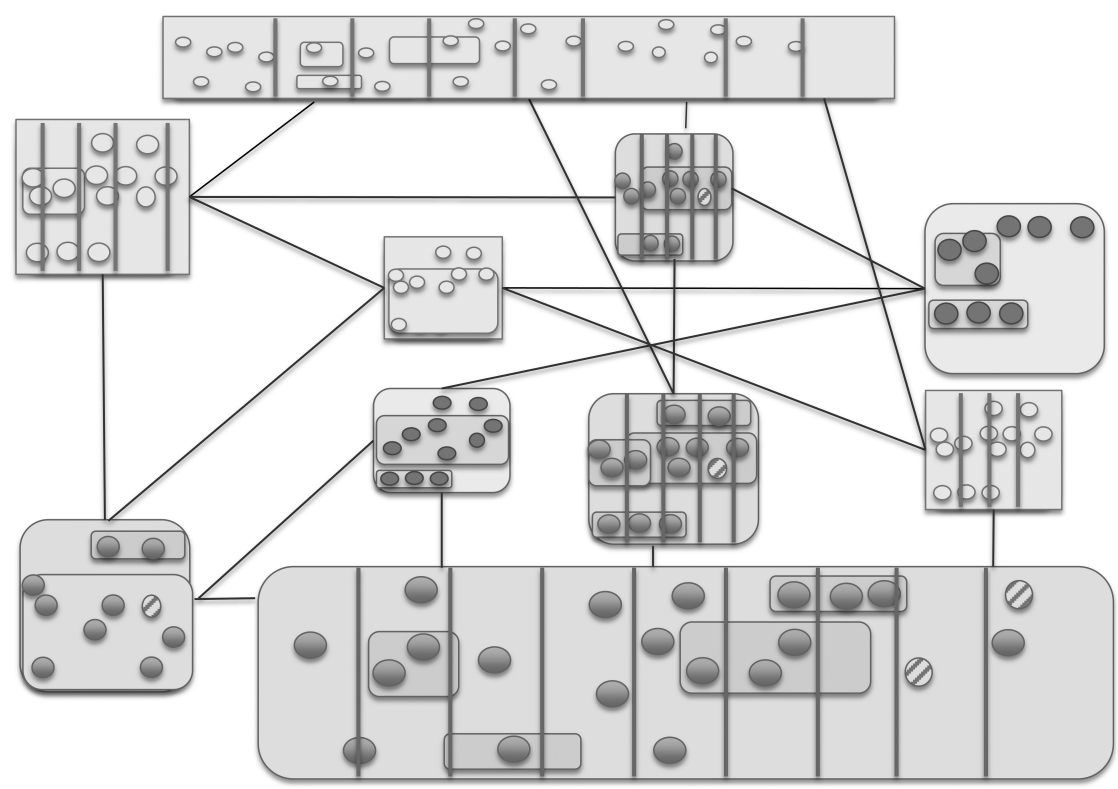

Figure 2.3 BOAP investigation designs several lines of sub-study of different aspects of sociotechnical change.

ethnographic adoption study that features an excess of observation units and data for answering whether or not a community or an organization has adopted/adapted a novelty system (Figure 2.2a) can be inadequate regarding the process of adoption if, for instance, observation units are missing data on some part of the process or if the data in many parts concerns, say, only one aspect of the process, such as how the technology is altered or how the users 
evaluate the technology (Figure 2.2b). Put methodologically, process questions require, by default, a series of observation units and typically also several analysis units on different kinds of events and phases in order to cover the relevant and typically interacting phenomena involved. (Figure 2.2c).

This then leads us to the rationale for BOAP studies: if one is to study change over time and beyond just a single organization, what kind of study set-up is required? The study design framing effects that BOAP is concerned with can remain systematically unrecognized. In diffusion studies, it took next to three decades to admit that diffusion surveys and the way in which diffusion research thought of the adoption process would not work for complex technology or for complex organizations (Attewell, 1992; McLaughlin et al., 1999; Rogers, 2010) and even fell short of understanding the cultural appropriation processes (Silverstone et al., 1992; Rogers, 2010).

When Pollock and Williams (2008) compared the findings from hundreds of packaged software implementation studies (typically conducted shortly after the implementation process with in-depth qualitative observation units and data sets) with hundreds of studies on software impact (conducted by asking the management survey questions later in the adoption process), the results were strikingly at odds: the latter produced an image of implementation as a productivity-enhancing improvement and the former portrayed it as productivitylowering turmoil in an organization. But when we compare the analysis units and observation units of these studies, we notice that the studies are not only asking different questions, using different theories, but were equally divorced with respect to when, how, and from what occupational groups their data of the "same" phenomenon is from (Figure 2.2d). To clarify the exact interrelation between the two types, comparative studies in the same project would need to reach the whole span of both studies, use both data gathering methods, and have the more detailed observation units' series for the whole process. This is not undoable but often rather tedious and thus not something that likely happens unless researchers are methodologically informed to do so.

Similar analysis unit, observation unit, and data issues apply to innovations. To cover more than the incidences of innovation by an organization, more numerous observation units would be needed, as was the case with organizational adoption. A major finding from the process studies of innovation has been that innovations are complex processes that tend to feature several follow-on innovations and reframings, span organizational boundaries, and have extended time frames-hence further complicating any attempt to inquire into their incidence using surveys and equally highlighting that the previous mainstream understanding of innovation, as borne out of studying how science was applied in corporate research environments, was largely an artifact of scant observation units and thin data that simply suppressed all the complexity and contingency of innovation from view (van de Ven, 1999; Russell and Williams, 2002; Poole and van de Ven, 2005; Garud and Gehman, 2012).

What BOAP brings into this research landscape is the realization that technologies evolve in organizations over several product generations, and so does 
their design, and so do the activities of various third-party actors in the market that shape both design and use. Previous innovation process studies had already shown that the phenomenon of innovation does not stop at the developer organization, but the BOAP findings underscore that it does not stop at the product launch or early-adopter sites either. The consequent further multiplication of the analysis units, observation units, and data presents a challenge to researchers. To study the process of innovation and sociotechnical change, we need to study the relevant set of locales and practices wherein the innovation is shaped (and conversely that are shaped by the innovation), hence the BOAP guideposts in the above section.

Given that innovation process studies, including BOAP studies, indicate time frames of years and decades for most innovation processes, scholars are faced with the sheer impossibility of, say, establishing a data track of videorecorded ethnography on all relevant observation units within all the needed analysis units in order to cover the span of times and sites within which an innovation is shaped. This entails a program to sample and compare insight from the different analysis units and observation units to understand innovation processes (Figure 2.3). As articulated in Sections 2.2 and 2.3, BOAP research designs rely on establishing arrays of analysis units and observation units with different levels of granularity. This zooming in and out is only possible when one has sufficiently rich and detailed data to do so. Figures 2.1 and 2.2 can be used to depict the situation. If one has Figure 2.2a- and 2.2b-type data for events A1 and A2, but Figure 2.1d-type data for event A3, one can use these to depict the events at the least-detailed level for A3-A1 and A2 are then just redacted to the same grain size to allow comparability, as if sieved through a "strainer" of the same coarseness (for a comparative biography study of data "sieving procedures," see Hyysalo et al., 2016c). However, if the data for A2 were in fact those of Figures $2.1 \mathrm{c}$ or $2.1 \mathrm{~b}$, its use in the same depiction becomes questionable. At best it can be treated as indirect evidence unless other data allows backing it up. The zooming out thus only becomes possible if one has enough factual reach and scope in the empirical data. In turn, zooming in is only possible when the density of observation units and data for these observations allows it.

\subsubsection{Theoretical case sampling and within-case sampling in the continuation of BOAP investigations}

The development of BOAP investigations may be more or less programmatic depending upon the availability of resources (e.g., staff time, the research funding environment) and access constraints. The beginnings of a BOAP investigation may not differ much from other innovation studies or STS research but, as the research progresses, previous research is extended to a string of further studies, building upon existing knowledge and the various ideas/issues that unfold from this work and reflecting upon puzzles and gaps in understanding 
and emerging theorizing. These continuation strategies can be discussed in terms of four types of theoretical sampling (Glaser \& Strauss, 1967).

The first type of theoretical sampling is the expansion of the units of analysis. This may arise because of multiple research concerns (such as the desire to cover internal variance within the field of study, addressing adjacent influences, or covering the more long-term dynamics such as the evolution of the studied technology and technology), or some other reason why a scholar would like to present a broader picture of one or several innovation processes. The ensuing questions typically concern which of the sites, times, and actors that influence the innovation currently remain as blanks or are suspiciously thinly covered. The theoretically sampling typically begins by re-examine the data one already has regarding the needed new observation/analysis units and, next, to assess if and what more data can be generated. This may mean another interview round, choosing some field-sites for further observation, data comparisons with peers, or devising a new research set-up for the upcoming years of research. A common strategy has also been to examine if prior studies could provide broader coverage from which one might zoom out. In the present book, this was done through following the Finnish heat-pump field for a decade while expanding the study focus in order to put the initial findings into a broader perspective.

The second theoretical sampling strategy is that of gradually shifting the focal perspective in follow-on studies so that they focus on new sets of relationships, locales, and types of actors identified as potentially relevant in previous studies. In the course of such a journey, both research questions and the relevant streams of literature are likely to change significantly. A good example is Pollock and William's $(2008,2016)$ focal shifts from studying the implementation of packaged software to their procurement, development, user groups, user communities, and industry analysts in order to gain a rounded understanding of the software's evolution. In the present study we moved from user-innovation in renewables to peer support, then to peer communities, onwards to usercreated information infrastructures and finally to user roles in affecting energy transition (Heiskanen et al., 2014; Hyysalo et al., 2013b, 2018).

The third theoretical sampling strategy concerns intensification through zooming in. Zooming in is typically motivated by a wish to understand a specific sub-phenomenon in a more nuanced way or to question an assumption made at a coarser level of depiction. Zooming in requires more dense data and is thus typically only possible to do in an uneven fashion. BOAP studies indicate that it is illusory to demand in-depth data on all sites and times of innovation but, equally, that one should not go about assuming that a given in-depth study would be the site where the interesting phenomenon happened unless one can show at a coarser level that this is probably the case. Hence the notion of "strategic ethnography" (Pollock and Williams, 2008) and a "focus on key junctions of change" (Hyysalo, 2004). In the present line of investigation several intensifications were pursued, for instance, intensifications to clarify the carbon footprint effects of user innovations (Mattinen et al., 2014; Heiskanen et al., 2015) and pathways to adopting S-RET equipment (Juntunen, 2014b). 
The fourth type of theoretical sampling is related to establishing focal interestcontext interrelations. Most BOAP studies focus primarily on a few interrelated aspects of innovation, such as in the present investigation role of users, the change in the artifacts, the shaping of the marketplace, and innovation communities. The rest of the innovation and underlying sociotechnical changes are typically examined more broadly as an enacted context for this focal interest and at a coarser level of depiction. The result is that research progresses through several parallel scales of inquiry ranging from the evolution of practices to episodic studies (of varying durations of minutes to months) (e.g., Hyysalo, 2010; Hyysalo and Usenyuk, 2015; Johnson, 2013).

Extending enquiry beyond single settings emphasizes the need for such extended theoretical sampling strategies in studying sociotechnical change. Different data types and sites of data collection have their own framing effects (Miettinen, 1993; Hyysalo, 2010; Murto et al., 2020a, 2020c). Ethnographic observation, recorded in field notes, and audio and video recordings, provides a first-hand experience of the realities of design and the use of technology. However, ethnographic understanding accumulates slowly and partially, and it is common that some processes may not-yet be readily recognized by the involved actors or research scholars and may only emerge over time or by contrasting different settings. Interviews provide a more focused method of eliciting knowledge but may be shaped by the interests and self-justification of the actors involved. The immediacy of the ethnographic insights that arise from field observation and interviews could bring to the surface particular conflicts, concerns, and events that appeared particularly interesting for research, and in this way they assist in analyzing other sources of data, such as documents (the effects of these BOAP theoretical sampiling strategies are schematically represented in Figure 2.3 study design - it is not a unified depiction but one that allows reasoning across different sites, aspects and granularities of analysis).

Similarly to other multi-site studies, BOAP research designs are built to allow for the further juxtaposition of different actors' narratives and perspectives and, in doing so, increase the trustworthiness and robustness of analysts' interpretations (Miettinen, 1993). The variety of the available data tends to grow with multi-sited and longitudinal investigations and allows both data and method triangulation (Denzin, 1989). The theoretical sampling strategies help to balance the framing effects in the accounts of the interaction created, and also, the extended scope of study tends to level out particular actor concerns, momentary hypes, or displays put on for the ethnographer when one enters the site over a sustained period.

Let us concretize this methodological treatise by briefly decomposing one well-known example and one recent case of a technology study with respect to the phenomenon, analysis units, observation units, and data in which we think BOAP sensitivity would lead to a significantly different interpretation of the study and the proposed concepts, and then we present and contrast this with the present study data sets and analyses. 
The study of agency in transition pathways by Geels et al. (2016) endorsed roughly the same ideas as those of BOAP: multiple layers of analysis, and zooming in and zooming out to study sociotechnical change. The study had twofold conceptual objectives. The first was to qualify Geels and Schot's (2007) concept of transition pathways by examining if the real-time transition pathways are as uniform as the long time frame historical case studies suggested and if they could, in fact, shift from one archetypical pathway to another. The second conceptual objective was to answer to the critique among technology studies scholars that the multi-level framework ignores the actor perspective and, in doing so, the enactment of sociotechnical change (e.g., Shove et al., 2012; Hyysalo, 2010; Spinardi \& Slayton, 2015). Empirically, the paper analyzes the UK's and Germany's renewable energy transition in 5-10 year phases, noting in each phase how the overall development had progressed and how each incumbent and renewable industry field and policy decision had changed it. The changes in the archetypical pathway types in the 20-year time span are then documented. The argument that the transition pathways, examined more in real time, are likely to be more complex is argued for convincingly. From a BOAP perspective, however, the ensuing first question becomes: would the earlier overall transition trajectories appear as uniform as originally depicted if historians had access to as detailed material available per five-year phase as that of the Geels et al. (2016) case? That is, are the neat overall trajectories in, for example, Geels and Schot (2007) mostly an artifact of scant data and thin analysis rather than that of rigorous theoretical abstraction? Regarding the second objective, Geels et al. (2016) noted that in their study they do not zoom in to study the enactment of change at policy setting or company level, and thus the "enactment" is discussed at the level of "nuclear operators," "the coal industry," "the wind energy sector," and "the government." In the BOAP view, this grain-sized data still remains at a scale where only the gross outcomes of enactments may be visible and where no such social actors exist to devise any actions and thus "enact" transition on the ground (cf. Garud \& Gehman, 2012). Not at all belittling the usefulness of the meso-perspective that Geels et al. (2016) pursue, an actor perspective would entail depicting how particular natural or corporate bodies — such as organizations, associations, collective actor groups, policymakers, and so on-would have perceived and sought to act in relation to the transition pathway (cf. Garud and Gehman, 2012; Höyssä and Hyysalo, 2009). The framing of the study in relation to its execution thus still appears, according to the BOAP perspective, as a lower-level systems view rather than that of an "actor perspective" on the transition, even if the analysts conducted more focused and real-time systems analysis. The positive message from BOAP would be that there may be a good possibility to next accumulate more detailed data sets in order to move into the study of the actual actor perspectives. With more fine-grained data, one could zoom in and out on the process of enactment and sociotechnical transition, and between the grain size of actor negotiations and contingencies, and the currently used countrylevel view of sociotechnical transition. The meso-level depiction Geels et al. 
2016 had achived would provide excellent contextualization for these studies. Hence, in the case of Geels et al. (2016), the empirical study may be well done, but its conceptual interpretation stretches beyond the evidence they have at hand as it tries to account for phenomena that are only ever likely to become visible once one further zooms-in to a still finer grain size.

The BOAP study in the present book presents such zooming to finer grainsizes and has been anchored to heat pumps in the residential housing sector in Finland. By anchoring I mean that all the sub-studies and analyses include an analysis of residential sector heat pumps, even though the innovation- and adoption-related sub-studies reach out to other S-RETs in Finland (pellet burners, solar collectors, and solar PV) and some of the study aspects make international comparisons in order to contextualize the innovation, diffusion, community, and transition phenomena investigated. The characterization of Finland with respect to S-RET developments and the specific data and analysis procedures are reported in the Appendix of this book.

In the next chapters we move onto examining the yield of the BOAP approach in order to understand the user activities in renewables innovation and transition, and return to reflect on this deployment of BOAP in the concluding chapter.

\section{Note}

1 The chapter introduction and Section 2.2 and 2.3 build on research that has previously appeared in Hyysalo, S., Pollock, N. and Williams, R., (2019a). "Method matters in the social study of technology: Investigating the biographies of artifacts and practices." Science and Technology Studies 32 (3) 2-25. 